\title{
Tomoscintigraphy Improves the Determination of the Embryologic Origin of Parathyroid Adenomas, Especially in Apparently Inferior Glands: Imaging Features and Surgical Implications
}

\author{
David Taïeb ${ }^{1}$, Rim Hassad ${ }^{1}$, Frédéric Sebag ${ }^{2}$, Cécile Colavolpe ${ }^{1}$, Eric Guedj ${ }^{1}$, Elif Hindié ${ }^{3}$, Jean-François Henry ${ }^{2}$, and \\ Olivier Mundler ${ }^{1}$ \\ ${ }^{1}$ Service Central de Biophysique et de Médecine Nucléaire, Centre Hospitalo-Universitaire de la Timone, Marseille, France; ${ }^{2}$ Service de \\ Chirurgie Générale et Endocrinienne, Centre Hospitalo-Universitaire de la Timone, Marseille, France; and ${ }^{3}$ Service de Médecine \\ Nucléaire, Centre Hospitalo-Universitaire Saint Louis, Paris, France
}

\begin{abstract}
Identification of the embryologic origin of hyperfunctioning parathyroid adenomas in primary hyperparathyroidism (PHPT) could determine the most suitable approach for minimally invasive surgery. The aim of this study was to prospectively evaluate the reliability of a new, combined protocol for the preoperative localization and determination of the embryologic origin of parathyroid adenomas. Methods: Anterior dual-isotope $\left({ }^{123} / /{ }^{99 m} T c-\right.$ sestamibi) static planar imaging followed by tomoscintigraphy (SPECT acquisition) centered over the $140-\mathrm{keV}$ photopeak (combined protocol) was performed on 35 consecutive patients with sporadic PHPT. On the basis of anatomic considerations, adenomas were classified as superior ( $\mathrm{P} 4$ derived) if they were located above the isthmus or posterior to the thyroid on SPECT images, despite their apparently middle to inferior position, and as inferior (P3 derived) if the foci were located in inferior and anterior positions or along the thyrothymic tract. Parathyroid ultrasonography was performed on all patients. Results: A total of 36 adenomas were removed: 34 solitary adenomas and 1 double adenoma (for totals of 19 P3-derived and 17 P4-derived adenomas). Pinhole subtraction imaging, SPECT, and ultrasonography sensitivities for detecting adenomas were $86 \%, 78 \%$, and $77 \%$, respectively. False-positive contralateral images were observed only with ultrasonography (3 cases). Positive SPECT results were associated with higher gland weights. Thirteen glands were identified by SPECT as posterior glands, despite their apparently inferior position, and were removed through an appropriate lateral endoscopic approach. Eleven (85\%) of these glands had a P4 origin. Only 2 corresponded to large P3-derived adenomas (>2 g). Conclusion: By reclassifying apparently inferior adenomas as P4derived adenomas prolapsed behind the thyroid gland, SPECT provides information about the most suitable surgical approach for avoiding recurrent laryngeal nerve injury. Additional pinhole images should increase the detection of small adenomas. The combined protocol offers both advantages.
\end{abstract}

\footnotetext{
Received Jan. 12, 2007; revision accepted Apr. 19, 2007.

For correspondence or reprints contact: David Taïeb, Service Central de Biophysique et de Médecine Nucléaire, Centre Hospitalo-Universitaire de la Timone, 264 Rue Saint-Pierre, 13385 Marseille Cedex 5, France. E-mail: david.taieb@ap-hm.fr

COPYRIGHT @ 2007 by the Society of Nuclear Medicine, Inc.
}

Key Words: hyperparathyroidism; subtraction imaging; SPECT; minimally invasive surgery

J Nucl Med Technol 2007; 35:135-139

DOI: 10.2967/jnmt.107.039743

$\mathbf{T}$ he diagnosis of primary hyperparathyroidism (PHPT) is based on laboratory criteria, including hypercalcemia, normal to elevated urinary calcium excretion, and inappropriately normal to elevated parathyroid hormone (PTH) levels. Surgery is the first-line therapy for PHPT despite the controversies that still exist regarding asymptomatic patients. A solitary parathyroid eutopic adenoma is the most common cause of PHPT, and preoperative imaging studies have only limited impact on bilateral cervicotomy (conventional open surgery) results (1). In contrast, identifying uniglandular disease has become crucial in selecting patients for minimally invasive surgery (MIS) (2). Parathyroid scintigraphy and neck ultrasonography are not reliable for identifying multiglandular disease, but their negative predictive value for multiglandular disease is high (3). Another aim of preoperative imaging is to localize as precisely as possible a hyperfunctioning gland. This is particularly true for parathyroid MIS, for which the accurate anatomic localization of a gland should also determine the most suitable surgical route for gland removal. At our institutions, the choice of the minimally invasive technique depends on the risk of recurrent laryngeal nerve injury during the dissection, which is dependent on the embryologic origin of the gland. Because the nerve runs posteriorly to the thyroid lobes, resections of P4-derived and, less frequently, posterior P3-derived adenomas are risky and should be performed via a lateral endoscopic route (4). In contrast, inferior glands should be removed via a small anterior incision.

Many studies have demonstrated the high sensitivity of parathyroid planar scintigraphy with 1 radiolabeled tracer 
(dual-phase protocol) or 2 radiolabeled tracers (subtraction method) for precisely locating the side and position of hyperfunctioning glands. The pinhole subtraction method seems to have better sensitivity than the dual-phase protocol for detecting small lesions or adenomas in patients with concomitant nodular thyroid disease. However, information provided by planar images is often insufficient to describe the embryologic origin of the involved glands. Such data are obtained by oblique pinhole imaging or SPECT acquisition. SPECT provides information on the neck and mediastinum. To our knowledge, the specific combination of dual-tracer pinhole subtraction imaging and SPECT has never been evaluated. The aim of this study was to evaluate a protocol involving high-resolution planar pinhole imaging followed by SPECT acquisition (combined protocol) for 35 consecutive patients who were good candidates for MIS.

\section{MATERIALS AND METHODS}

\section{Patients}

Between May 2006 and September 2006, 35 consecutive patients with laboratory evidence of PHPT were enrolled in the study. Inclusion criteria were as follows: apparent sporadic disease (absence of other endocrine tumor, negative family history), absence of a previous history of thyroidectomy, and absence of thyroid disease that warranted a transverse cervicotomy approach. Thyroid disease was excluded on the basis of clinical history, palpation, and available imaging data.

Preoperative imaging included neck ultrasonography and parathyroid scintigraphy. According to the results of the imaging workup, patients underwent surgery via a minimally invasive approach or a conventional bilateral cervicotomy. For patients undergoing MIS, the success of the surgery was validated by measurement of intraoperative PTH levels (50\% decrease compared with the highest preexcision value). Cure was defined as normalization of serum calcium and PTH levels after gland removal.

\section{Parathyroid Scintigraphy Combined Protocol}

All patients underwent dual-isotope $\left({ }^{123} \mathrm{I} /{ }^{99 \mathrm{~m}} \mathrm{Tc}\right.$-sestamibi) static planar pinhole imaging followed by ${ }^{99 \mathrm{~m} T c-s e s t a m i b i}$ SPECT on the same day. The same camera (Siemens ECAM) was used for both image acquisitions, but equipped, respectively, with pinhole and parallel-hole, low-energy, high-resolution collimators. The steps of the protocol were as follows.

First, the patients received $12 \mathrm{MBq}$ of ${ }^{123} \mathrm{I}$. Two hours later, 740 $\mathrm{MBq}$ of ${ }^{99 \mathrm{~m}} \mathrm{Tc}$-sestamibi were injected. Pinhole acquisition was started $3 \mathrm{~min}$ after ${ }^{99 \mathrm{~m}} \mathrm{Tc}$-sestamibi injection; static anterior cervical views were obtained for 20 min with 2 photopeaks of $7 \%$ energy windows, centered, respectively, over the $140-\mathrm{keV}$ photopeak of ${ }^{99 \mathrm{~m}} \mathrm{Tc}$ and over the $159-\mathrm{keV}$ photopeak of ${ }^{123} \mathrm{I}$ (128 $\times 128$ matrix, zoom of 2.67). Interactive software was used for image normalization and subtraction.

The tomographic study was started after the collimators were changed (at 30-45 min after sestamibi injection), with the following parameters: $128 \times 128$ matrix, zoom of $1.45,45 \mathrm{~s}$ per projection at each of 32 angular steps, and sampling of a $360^{\circ}$ orbit every $24^{\circ}$. The photopeak was centered over $140 \mathrm{keV}$ only, and iterative reconstruction was performed with a gaussian filter (full width at half maximum, $10 \mathrm{~mm}$ ). The neck and the mediastinum (from the angle of the mandible to the heart) were included in the field of view.

\section{Image Interpretation}

Two independent nuclear medicine physicians reviewed pinhole subtraction and SPECT images separately.

A positive finding on pinhole images was defined as residual ${ }^{99 \mathrm{~m}} \mathrm{Tc}$ activity after normalization and subtraction. On SPECT images, a positive finding was defined as focal uptake in an ectopic position (posterior or inferior to the thyroid lobe, mediastinal) or as focal tracer retention in an area of the thyroid distinct from the remaining thyroid tissue. Lesions were classified as superior ( $\mathrm{P} 4$ derived) if they were located above the isthmus or behind the thyroid on SPECT images, despite their apparently inferior position, and as inferior ( $\mathrm{P} 3$ derived) if they were located in inferior and anterior glands (at the tip of the inferior pole of the thyroid lobe or along the thyrothymic tract).

Finally, a finding was designated as positive if both reviewers were in agreement. In case of disagreement, a third reviewer was used to reach a consensus.

\section{Additional Imaging Techniques}

The same trained radiologist performed neck ultrasonography on all patients (Philips-ATL HDI 5000 system with a $12-\mathrm{MHz}$ linear transducer).

\section{Statistical Analysis}

To identify factors that could influence imaging results, distributions of age, serum calcium levels, PTH levels, and gland weight in patients with positive and negative results were compared with a Mann-Whitney-Wilcoxon test. In patients with a solitary adenoma, the correlation among preoperative serum calcium levels, PTH levels, and gland weight was calculated with the Pearson correlation coefficient.

\section{RESULTS}

\section{Patient and Tumor Characteristics}

Thirty of the 35 patients were women, with a mean age of $61 \mathrm{y}$ (mean $\pm \mathrm{SD}, 61 \pm 11 \mathrm{y}$ ). Preoperative median serum calcium and PTH levels were $2.65 \mathrm{mmol} / \mathrm{L}(2.69 \pm$ $0.13)$ and $79 \mathrm{ng} / \mathrm{mL}(97 \pm 71)$, respectively. A total of 36 adenomas were removed: 34 solitary adenomas and 1 double adenoma (left and right P4-derived adenomas). The median gland weight was $880 \mathrm{mg}(1,095 \pm 880)$. Gland weight was significantly correlated with preoperative PTH levels $\left(P=0.047, r^{2}=0.34\right)$ but not with hypercalcemia. Only one supernumerary ectopic gland, located in the thymus, was identified (130 mg). No major ectopia was found. Adenomas developed from P3 glands in 19 patients (6 right P3-derived and 13 left P3-derived adenomas) and P4 glands in 16 patients (17 adenomas: 11 right P4-derived and 6 left P4-derived adenomas, including 1 P4-derived double adenoma).

\section{Imaging Results and Surgical Considerations}

Diagnostic Accuracy of Parathyroid Scintigraphy and Intrapatient Comparison of Protocols. Planar pinhole imaging results were positive for 31 of 36 adenomas $(86 \%)$. SPECT results were positive for 28 of 36 adenomas $(78 \%)$. No false-positive lesion was reported. In 3 cases, adenomas 
were visualized only on pinhole images $(130,200$, and 580 $\mathrm{mg}$ ). No lesion was localized by SPECT alone. The double adenoma was visualized by both scintigraphy protocols. In 2 cases, the localization of adenomas was doubtful only on SPECT images and required a third reviewer to reach a consensus. Gland weight was significantly higher in patients with positive SPECT results $(P=0.0078)$ than in those with negative results. Age, serum calcium levels, and PTH levels were not statistically different for positive and negative pinhole subtraction imaging and SPECT results.

Comparison of Parathyroid Scintigraphy and Ultrasonography. Parathyroid ultrasonography correctly identified 28 adenomas (78\%) but produced contralateral false-positive results in 3 cases. Ultrasonography provided the sole positive results in 2 cases ( 350 and $680 \mathrm{mg}$ ). It missed one lesion in the patient with a double adenoma.

Value of SPECT Used with Planar Imaging for Determination of Embryologic Origin of Involved Glands and Surgical Implications. As shown in Figure 1, P4-derived adenomas seen on planar images were widely distributed throughout the inner part of the thyroid lobe. Half of the P4-derived adenomas were located in the middle portion of the thyroid lobe, at the medial margin, close to the isthmus. In contrast, $95 \%$ of P3-derived adenomas were located at the tip of the inferior lobe or along the thyrothymic tract. Thirteen glands were identified by SPECT as posterior glands, despite their apparently inferior position, and were removed through an appropriate lateral endoscopic approach. Eleven $(85 \%)$ of these glands had a P4 origin. Only 2 corresponded to large P3-derived adenomas ( $>2 \mathrm{~g})$. By reclassifying apparently inferior adenomas as $\mathrm{P} 4$-derived adenomas prolapsed behind the thyroid gland, SPECT provided information about the most suitable surgical approach for avoiding recurrent laryngeal nerve injury (Figs. 2-4). Anterior P3-derived adenomas were removed via a small cervical incision (mini-opening approach). Patients with negative preoperative imaging results, discordant imaging results, or multiglandular disease underwent conventional bilateral cervical surgery. Thus, 29 patients underwent surgery via a focused approach, and 6 underwent conventional bilateral cervical surgery. No surgical conversion was performed. Only one patient had persistent hyperparathyroidism because of partial gland removal. Other patients had normal serum calcium and PTH levels. No hypoparathyroidism or recurrent nerve palsy was observed.

\section{DISCUSSION}

Since the introduction of focused surgical approaches for parathyroid surgery, preoperative parathyroid scintigraphy has played an increasing role in the preoperative workup for patients with PHPT. The combined protocol has a high sensitivity (86\%) and provides useful information for MIS, such as the anteroposterior position of the hyperfunctioning glands in the neck and the mediastinum, which is closely related to the embryologic origin of the involved glands. The overall duration of camera use with the combined protocol is about $1 \mathrm{~h}$, including time needed to change collimators. The low rate of multiglandular disease (1/35 patients) and the absence of major ectopia in the present study are compatible with traditional findings for patients selected for MIS.

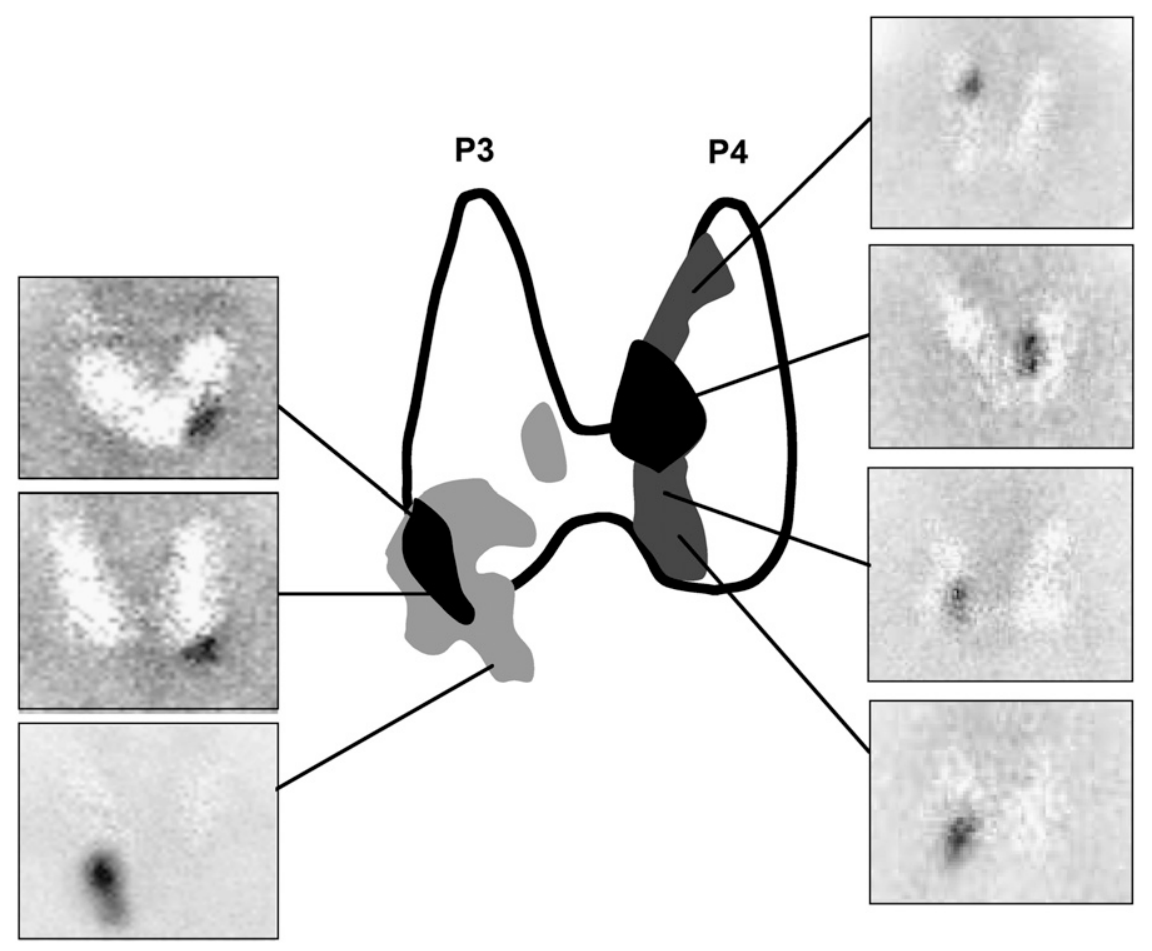

FIGURE 1. Projection of 31 parathyroid adenomas on planar pinhole images. Half of P4-derived adenomas were located in midportion of thyroid lobe, at medial margin, close to isthmus. In contrast, 95\% of P3-derived adenomas were located at tip of inferior lobe or along thyrothymic tract. Most frequent sites are represented by shading. Note that there are potential areas of overlap between P3- and P4-derived adenomas. 


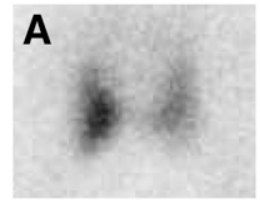

B
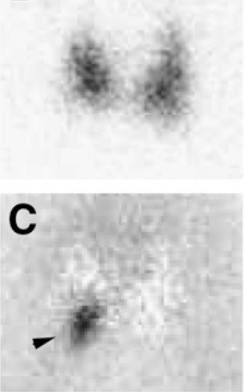

D

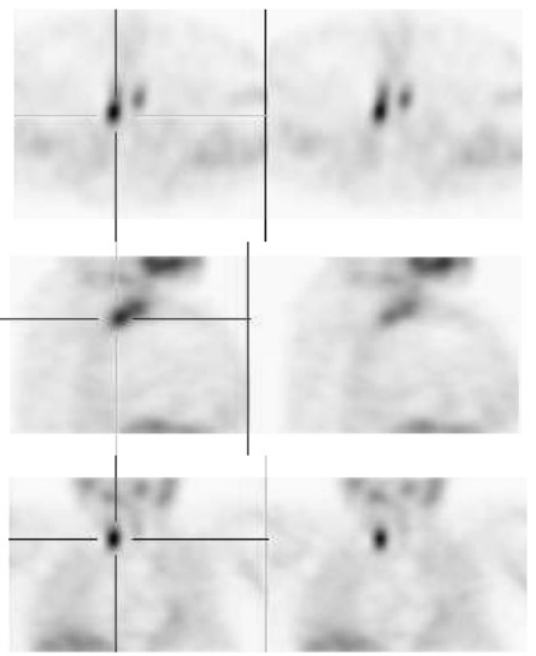

FIGURE 2. Planar pinhole images (A: 99mTc-sestamibi; B: ${ }^{123}$; C: subtraction) and SPECT images (D) of parathyroid adenomas. Posterior extension of adenoma on SPECT images is highly suggestive of $\mathrm{P} 4$ origin, despite its apparently right inferior origin on planar images (arrowhead).

The sensitivity of pinhole subtraction imaging in the present study was comparable to that in the literature (5). Reported sensitivities of parathyroid scintigraphy for detecting adenomas were excellent for both subtraction and dualphase protocols, ranging from $70 \%$ to $100 \%(3,5-9)$. Gland weight and preoperative serum PTH levels were reported to be the more important factors affecting the detection of hyperfunctioning parathyroid glands. These data account for the apparent discrepancies in sensitivities and make difficult the comparison of imaging protocols among series, which included sporadic disease, multiple endocrine neoplasia-related hyperplasia, and recurrences. In the pre-
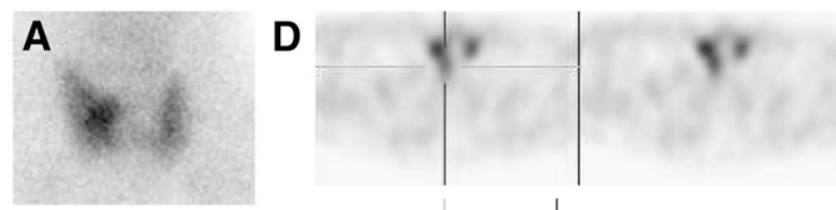

B

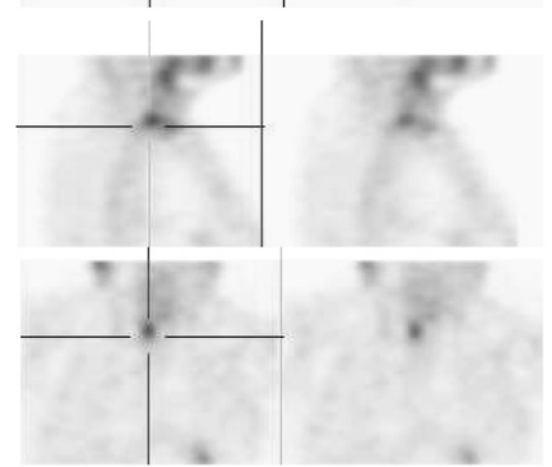

FIGURE 3. Planar pinhole images (A: 99mTc-sestamibi; B: ${ }^{123}$; C: subtraction) and SPECT images (D) of parathyroid adenomas. Adenoma is located at posteromedial part of right thyroid lobe (arrowhead), above isthmus; this location is characteristic of P4 origin.
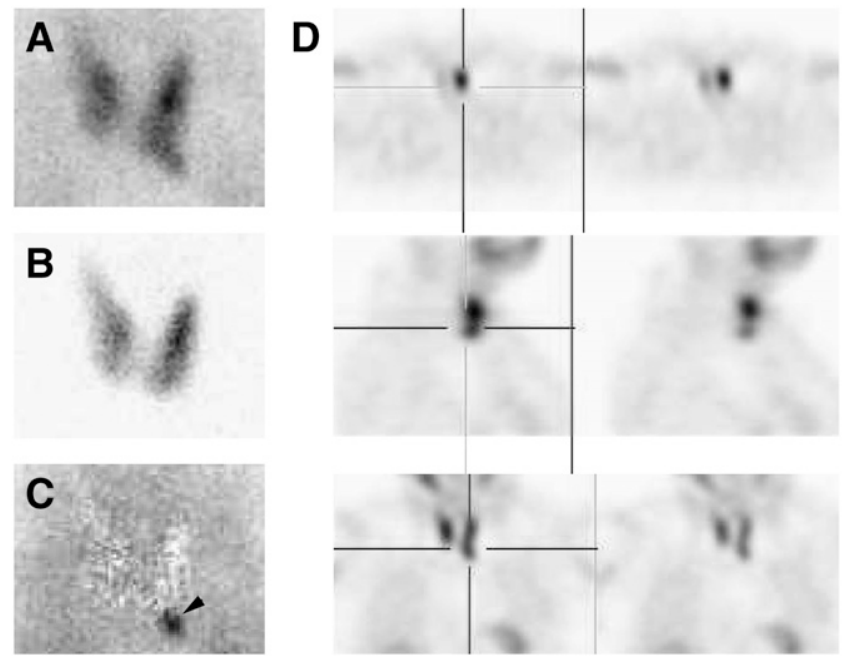

FIGURE 4. Planar pinhole images (A: 99mTc-sestamibi; B: ${ }^{123}$; C: subtraction) and SPECT images (D) of parathyroid adenomas. Typical P3 adenoma is located at tip of left inferior lobe on planar images (arrowhead) and in anterior position on SPECT images.

sent study, positive SPECT results were associated with higher gland weights. The subtraction method seemed to yield better results than dual-phase imaging, despite the fact that only a few studies included an intrapatient comparison analysis. Leslie et al. (5) compared ${ }^{99 \mathrm{~m}} \mathrm{Tc}$-pertechnetate/99m Tc-sestamibi subtraction images and delayed planar images (at $2 \mathrm{~h}$ ) in an intrapatient analysis. Sixtyeight patients had a solitary adenoma. The gland weight was $1,668 \pm 2,241 \mathrm{mg}$ (mean $\pm \mathrm{SD}$ ). The sensitivities of subtraction imaging only and sestamibi imaging only were $85 \%$ and $90 \%$, respectively. The missed gland weights were not statistically different from those of the detected adenomas (5). Hindié et al. (6) studied 27 cases of sporadic PHPT with a ${ }^{123} \mathrm{I} /{ }^{99 \mathrm{~m}} \mathrm{Tc}$-sestamibi pinhole subtraction method. Twenty-six of 27 solitary adenomas were correctly identified, and one false-positive uptake result was attributed to a second parathyroid lesion. In that study, the median gland weight was $923 \mathrm{mg}$ (mean $\pm \mathrm{SD}, 1,336 \pm 1,461$ ), and the median PTH level was $121 \mathrm{ng} / \mathrm{L}(145 \pm 74)$. The missed gland weighed $126 \mathrm{mg}(6)$.

Several studies have demonstrated the role of SPECT acquisition in improving the localization of adenomas in patients undergoing surgery via minimally invasive approaches. Moka et al. (7) studied 92 patients with ${ }^{99 \mathrm{~m} T c-}$ pertechnetate $/ 99 \mathrm{~m} \mathrm{Tc}$-sestamibi subtraction and SPECT imaging (at $2 \mathrm{~h}$ after injection). The adenomas weighed between 95 and 1,000 $\mathrm{mg}(440 \pm 220)$. There were 7 cases of double adenoma and 3 cases of mediastinal ectopia. SPECT was superior to planar imaging in 7 cases (sensitivity, $95 \%$ vs. $87 \%$ ) (7). In the study of Civelek et al. (8), more than one fourth of lesions found positive by sestamibi SPECT weighed less than or equal to $500 \mathrm{mg}$. A $60-\mathrm{mg}$ adenoma was correctly identified. However, the use of delayed SPECT alone led to a large number of false-positive 
readings (8). Lorberboym et al. (9) studied 52 patients with PHPT by using a dual-phase planar protocol, SPECT acquisition (at $20 \mathrm{~min}$ after injection) and planar subtraction imaging. Early sestamibi SPECT revealed 9 more adenomas than combined planar techniques (sensitivity, $96 \%$ vs. $76 \%$ ) in 6 patients with multinodular goiters and 3 patients with ectopic glands. The average adenoma weight was $1.14 \mathrm{~g}(0.170-4)(9)$. In the study of Gayed et al. (10), SPECT images (at $30 \mathrm{~min}$ after injection) provided information beyond that provided by dual-phase planar images for gland localization, and image interpretation changed for up to $33 \%$ of images. CT was particularly helpful for ectopic glands. Among the 32 patients undergoing surgery, 29 had an adenoma. In the 3 remaining patients, with double lesions, SPECT identified only one hyperfunctioning gland. In that study, the preoperative PTH levels were not significantly different between patients with positive scans and patients with negative scans (10). In a recent study carried out by Rubello et al. (11), SPECT correctly identified 4 adenomas that were located deep in the paraesophageal and paratracheal spaces from among the 22 solitary lesions removed by radiologically guided minimally invasive surgery. The gland weight for these 22 adenomas was $1,098 \pm 378 \mathrm{mg}$ (11). In our study, the lower SPECT sensitivity could have been related to the protocol. The massive dose difference between the radiotracers avoids significant contamination from ${ }^{123} \mathrm{I}$ to ${ }^{99 \mathrm{~m}} \mathrm{Tc}$ on SPECT images. However, it is possible that delayed SPECT images obtained after the injection of sestamibi alone have a higher signal-to-noise ratio because of thyroid washout.

Acquisitions could also have taken place too early for optimal ${ }^{99 \mathrm{~m}}$ Tc-sestamibi retention. When a single tracer is used, delayed SPECT acquisition is associated with a better parathyroid-to-thyroid ratio; however, in our combined protocol, it could lead to a decreased accumulation of sestamibi in parathyroid adenomas (because of sestamibi washout) but to a significant persistence of ${ }^{123}$ I retention in the thyroid gland (because of iodide organification).

In our opinion, SPECT should not be used to replace planar imaging because pinhole magnification is a very sensitive method for small embedded lesions in the thyroid lobes and the images were more convincing for independent reviewers (they always agreed). Furthermore, SPECT alone was associated with more false-positive foci in previous series.
The proposed combination protocol has high sensitivity and provides useful 3-dimensional information for surgeons who perform MIS, with a good compromise between time spent and effectiveness in comparison with the data for published protocols. The anteroposterior description of an adenoma (related to the embryologic origin) allows the surgeon to choose the most suitable surgical approach to avoid injury to the recurrent laryngeal nerve, which is exposed during the resection of superior and posterior adenomas (mainly of P4 origin). Additional pinhole images should increase the detection of small adenomas.

\section{CONCLUSION}

The combination of dual-tracer planar pinhole scintigraphy and SPECT acquisition is a promising tool for choosing the optimal minimally invasive technique.

\section{REFERENCES}

1. Allendorf J, Kim L, Chabot J, DiGiorgi M, Spanknebel K, LoGerfo P. The impact of sestamibi scanning on the outcome of parathyroid surgery. J Clin Endocrinol Metab. 2003;88:3015-3018.

2. Carty SE, Worsey J, Virji MA, Brown ML, Watson CG. Concise parathyroidectomy: the impact of preoperative SPECT ${ }^{99 \mathrm{~m}} \mathrm{Tc}$ sestamibi scanning and intraoperative quick parathormone assay. Surgery. 1997;122:1107-1114.

3. Sebag F, Hubbard JG, Maweja S, Misso C, Tardivet L, Henry JF. Negative preoperative localization studies are highly predictive of multiglandular disease in sporadic primary hyperparathyroidism. Surgery. 2003;134:1038-1041.

4. Henry JF, Sebag F, Tamagnini P, Forman C, Silaghi H. Endoscopic parathyroid surgery: results of 365 consecutive procedures. World J Surg. 2004;28:12191223 .

5. Leslie WD, Dupont JO, Bybel B, Riese KT. Parathyroid ${ }^{99 \mathrm{~m}} \mathrm{Tc}$-sestamibi scintigraphy: dual-tracer subtraction is superior to double-phase washout. Eur $J$ Nucl Med Mol Imaging. 2002;29:1566-1570.

6. Hindié E, Melliere D, Simon D, Perlemuter L, Galle P. Primary hyperparathyroidism: is technetium $99 \mathrm{~m}$-sestamibi/iodine-123 subtraction scanning the best procedure to locate enlarged glands before surgery? J Clin Endocrinol Metab. 1995;80:302-307.

7. Moka D, Voth E, Dietlein M, Larena-Avellaneda A, Schicha H. Technetium 99m-MIBI-SPECT: a highly sensitive diagnostic tool for localization of parathyroid adenomas. Surgery. 2000;128:29-35.

8. Civelek AC, Ozalp E, Donovan P, Udelsman R. Prospective evaluation of delayed technetium-99m sestamibi SPECT scintigraphy for preoperative localization of primary hyperparathyroidism. Surgery. 2002;131:149-157.

9. Lorberboym M, Minski I, Macadziob S, Nikolov G, Schachter P. Incremental diagnostic value of preoperative ${ }^{99 \mathrm{~m}}$ Tc-MIBI SPECT in patients with a parathyroid adenoma. J Nucl Med. 2003;44:904-908.

10. Gayed IW, Kim EE, Broussard WF, et al. The value of ${ }^{99 \mathrm{~m} T c-s e s t a m i b i}$ SPECT/ CT over conventional SPECT in the evaluation of parathyroid adenomas or hyperplasia. J Nucl Med. 2005;46:248-252.

11. Rubello D, Massaro A, Cittadin S, et al. Role of ${ }^{99 \mathrm{~m}} \mathrm{Tc}$-sestamibi SPECT in accurate selection of primary hyperparathyroid patients for minimally invasive radio-guided surgery. Eur J Nucl Med Mol Imaging. 2006;33:1091-1094. 